\title{
Predictive factors for unfavorable outcomes of tuberculous pericarditis in human immunodeficiency virus-uninfected patients in an intermediate tuberculosis burden country
}

In Young Jung, Young Goo Song, Jun Yong Choi, Moo Hyun Kim, Woo Yong Jeong, Dong Hyun Oh, Yong Chan Kim, Je Eun Song, Eun Jin Kim, Ji Un Lee, Su Jin Jeong ${ }^{*}$, Nam Su Ku and June Myung Kim

\begin{abstract}
Background: In areas where Mycobacterium tuberculosis is endemic, tuberculosis is known to be the most common cause of pericarditis. However, the difficulty in diagnosis may lead to late complications such as constrictive pericarditis and increased mortality. Therefore, identification of patients at a high risk for poor prognosis, and prompt initiation of treatment are important in the outcome of TB pericarditis. The aim of this study is to identify the predictive factors for unfavorable outcomes of TB pericarditis in HIV-uninfected persons in an intermediate tuberculosis burden country.

Methods: A retrospective review of 87 cases of TB pericarditis diagnosed at a tertiary referral hospital in South Korea was performed. Clinical characteristics, treatment outcomes, complications during treatment, duration of treatment, and medication history were reviewed. Unfavorable outcome was defined as constrictive pericarditis identified on echocardiography performed 3 to 6 months after initial diagnosis of TB pericarditis, cardiac tamponade requiring emergency pericardiocentesis, or death. Predictive factors for unfavorable outcomes were identified.

Results: Of the 87 patients, 44 (50.6\%) had unfavorable outcomes; cardiac tamponade $(n=36)$, constrictive pericarditis $(n=18)$, and mortality $(n=4) .14$ patients experienced both cardiac tamponade and constrictive pericarditis. During a 1 year out-patient clinic follow up, 4 patients required repeat pericardiocentesis and pericardiectomy was performed in 0 patients. In the multivariate analysis, patients with large amounts of pericardial effusion $(P=.003)$, those with hypoalbuminemia $(P=.011)$, and those without cardiovascular disease $(P=.011)$ were found to have a higher risk of unfavorable outcomes.
\end{abstract}

Conclusion: HIV-uninfected patients with TB pericarditis are at a higher risk for unfavorable outcomes when presenting with low serum albumin, with large pericardial effusions, and without cardiovascular disease.

Keywords: Tuberculous pericarditis, Non-HIV, Unfavorable outcome

\footnotetext{
*Correspondence: JSJ@yuhs.ac

Department of Internal Medicine and AIDS Research Institute, Yonsei

University College of Medicine, 50-1 Yonsei-ro, Seodaemun-gu 120-752,

Seoul, Republic of Korea
} 


\section{Background}

Tuberculosis is a globally important infectious disease with a high mortality and morbidity, with 1.5 million deaths among the human immunodeficiency virus (HIV)-negative and 0.52 million deaths among the HIV-positive population, and with 9.4 million new cases reported each year worldwide in 2014 [1]. In areas where Mycobacterium tuberculosis is endemic, tuberculosis is known to be the most common cause of pericarditis [2]. Although the incidence of tuberculosis has decreased in many Western countries, tuberculous (TB) pericarditis is still seen in many patients in countries where tuberculosis is still an endemic disease [3]. According to a study conducted in Tanzania, TB pericarditis is also more likely to occur in HIV-infected patients, whereas HIV-uninfected patients are likely to have other etiologies of pericardial effusions [4].

Symptoms of TB pericarditis are generally nonspecific, with an insidious onset [5]. The frequency of common symptoms varies; however, cough, dyspnea, chest pain, night sweat, orthopnea, and weight loss are generally the most frequent symptoms $[6,7]$. The diagnosis of TB pericarditis can be made through the identification of the tubercle bacillus from pericardial fluid; however, invasive diagnostic pericardial biopsy is not an easy process to perform [8]. Anti-TB therapy is effective in reducing mortality among HIV-uninfected persons to $8-17 \%$ [9]. A retrospective study conducted in Birmingham showed that antituberculosis medication reduced the likelihood of developing constrictive pericarditis to $10-20 \%$ [7]. However, the difficulty in diagnosis may lead to late complications such as constrictive pericarditis and increased mortality [5]. Therefore, identification of patients at a high risk for poor prognosis, and prompt initiation of treatment are important in the outcome of TB pericarditis.

We conducted this study to identify the predictive factors for the unfavorable outcomes of $\mathrm{TB}$ pericarditis in HIV-uninfected patients in an intermediate tuberculosis burden country.

\section{Methods}

\section{Study population}

We studied 166 patients with TB pericarditis diagnosed at a tertiary referral hospital in South Korea during an 11-year period (January 2005 through December 2015). Seventy-nine patients with diagnostic error $(n=11)$, missing laboratory data $(n=60)$, loss to treatment follow-up $(n=7)$, or concomitant HIV infection $(n=1)$ were excluded. A total of 87 patients were reviewed. Patients who were 18 years old or older who had been confirmed to have probable or definite TB pericarditis were included.

\section{Collected data}

The following information were collected: demographic data, comorbidities, initial presenting symptoms, laboratory results, echocardiographic findings, pericardial biopsy results, pericardial fluid smear microscopy for acid fast bacilli, pericardial culture for $M$. tuberculosis, antimicrobial susceptibility of $M$. tuberculosis strains isolated from the patients, pharmaceutical formulation use, steroid use, and duration of treatment. Patient follow-up was based on out-patient clinical visits.

\section{Definitions}

Definite TB pericarditis was defined as a diagnosis confirmed on a pericardial sample based on finding acid and alcohol fast bacilli on microscopy, positive microbiological culture for $M$. tuberculosis, presence of caseating granulomata on histology, or positive nucleic acid test (fluid or tissue) [10].

Probable TB pericarditis was defined as the presence of a lymphocytic pericardial exudate with elevated adenosine deaminase (ADA) activity $\geq 40$ IU/L [10]. Detailed information on definitions of definite and probable TB pericarditis is provided in Additional file 1: Table S1.

Unfavorable outcome was defined as constrictive pericarditis identified on echocardiography performed 3 to 6 months after initial diagnosis of TB pericarditis, cardiac tamponade requiring emergency pericardiocentesis, or death [10].

Cardiovascular disease was defined in patients with a history of hypertension, stable angina, and congestive heart failure. The amount of pericardial effusion was categorized into small (50-100 mL), moderate (100$500 \mathrm{~mL})$, or large $(>500 \mathrm{~mL})$ [11]. QuantiFERON-TB Gold In-Tube assay was used for interferon-gamma $($ IFN- $\gamma)$ release assay (IGRA) diagnosis. The IFN- $\gamma$ levels $(\mathrm{IU} / \mathrm{mL})$ were measured, and the results were reported as positive, negative, or indeterminate. Hypoalbuminemia was defined as serum albumin $<3.0 \mathrm{~g} / \mathrm{dL}$. Body mass index was calculated as the weight in kilograms divided by the square of height in meters [12]. Red blood cell $(\mathrm{RBC})$ distribution width was defined as the index of $\mathrm{RBC}$ size heterogeneity and $\mathrm{RBC}$ distribution width [13].

\section{Statistical analysis}

All factors were compared between the unfavorable and favorable outcome groups. Categorical variables are presented as numbers and percentages. Continuous variables are expressed as mean \pm standard deviation, unless otherwise indicated. Categorical variables were compared by using $X^{2}$ analysis, and continuous variables with normal distributions were compared by using Student's $t$-test. We used the Mann-Whitney $U$-test to compare the continuous variables with a skewed distribution. Multivariate analysis was performed through logistic regression to identify factors that independently and significantly affected the outcome. All categories (large 
pericardial effusions, dyspnea, male sex, albumin, and cardiovascular disease) were calculated as a percentage with the 95\% confidence interval (CI). All statistical tests were performed by using IBM SPSS software for Windows, version 20. $P$-values $<0.05$ were considered statistically significant.

\section{Results}

As shown in Table 1, of the 87 cases reviewed, 44 (50.6\%) patients had unfavorable outcome. Of these patients, 36 patients received pericardial effusion drainage due to cardiac tamponade, 18 patients had constrictive pericarditis, and mortality in 4 patients. 14 patients experienced both cardiac tamponade and constrictive pericarditis. During a 1 year out-patient clinic follow-up, 4 patients required repeat pericardiocentesis, and pericardiectomy was performed in 0 patients. The causes of mortality were acute heart failure due to stress-induced cardiomyopathy, and cardiac tamponade.

There was a male predominance in patients with unfavorable outcome compared with those with favorable outcome $(56.8 \%$ vs. $34.9 \%, P=.040)$. Other baseline characteristics of underlying diseases such as solid organ cancer, hematologic malignancies, hypothyroidism, old pulmonary tuberculosis, chronic renal disease, chronic liver disease, chronic lung disease, autoimmune disease, and diabetes mellitus did not show any difference between outcomes. On the other hand, patients without a history of cardiovascular disease were found to have a higher risk of unfavorable outcomes $(34.1 \%$ vs. $55.8 \%$, $P=.042$ )

Table 1 shows the laboratory results of the study participants at the time of admission. Patients presenting with a low albumin level at the time of admission tended to have unfavorable outcomes $(3.3 \pm 0.7$ vs. $3.6 \pm 0.6$, $P=.047$ ). Hypoalbuminemia defined as serum albumin lower than $3.0 \mathrm{~g} / \mathrm{dL}$ had higher risk of unfavourable outcome (38.6\% vs. $16.3 \%, P=.020)$. Pericardial ADA levels did not show a statistical significance between the two groups ( 85 vs. $63, P=.986$ ).

Table 1 describes the clinical symptoms and findings of the study participants. Dyspnea was the most common presenting symptom, and the presence of dyspnea showed a correlation to a higher risk of unfavorable outcomes $(84.1 \%$ vs. $62.8 \%, P=.024)$. Patients who presented with large amounts of pericardial effusion, exceeding $500 \mathrm{~mL}$ on the echocardiogram, showed a higher risk for unfavorable outcomes $(79.5 \%$ vs. $53.5 \%$, $P=.010)$. Concomitant pulmonary tuberculosis was seen in 6 (14\%) patients with favorable outcomes and 4 (9.1\%) patients with unfavorable outcomes, with no significant difference $(P=.521)$. The mean duration of symptom onset was 7 days in the favorable group and 14 days in the unfavorable group, showing that patients
Table 1 The baseline characteristics of the patients

\begin{tabular}{|c|c|c|c|}
\hline Characteristic & $\begin{array}{l}\text { Favorable } \\
(n=43)\end{array}$ & $\begin{array}{l}\text { Unfavorable } \\
(n=44)\end{array}$ & $P$-value \\
\hline Age (years) & $66.5 \pm 18.2$ & $67.1 \pm 15.5$ & $.873^{\mathrm{a}}$ \\
\hline Male, n (\%) & $15(34.9)$ & $25(56.8)$ & $.040^{\mathrm{b}}$ \\
\hline BMI & $22.8 \pm 4.9$ & $22.9 \pm 3.2$ & $.871^{\mathrm{a}}$ \\
\hline \multicolumn{4}{|l|}{ Underlying diseases, yes (\%) } \\
\hline Solid cancer & $2(4.7)$ & $4(9.1)$ & $.676^{\mathrm{b}}$ \\
\hline Hematologic malignancy & $0(0.0)$ & $3(6.8)$ & $.241^{\mathrm{b}}$ \\
\hline Hypothyroidism & $0(0.0)$ & $2(4.5)$ & $.504^{\mathrm{b}}$ \\
\hline Cardiovascular disease & $24(55.8)$ & $15(34.1)$ & $.042^{\mathrm{b}}$ \\
\hline Old tuberculosis & $6(14.0)$ & $8(18.2)$ & $.592^{\mathrm{b}}$ \\
\hline Chronic renal disease & $4(9.3)$ & $1(2.3)$ & $.202^{\mathrm{b}}$ \\
\hline Chronic liver disease & $0(0.0)$ & $3(6.8)$ & $.241^{\mathrm{b}}$ \\
\hline Chronic lung disease & $3(7.0)$ & $5(11.4)$ & $.713^{\mathrm{b}}$ \\
\hline Autoimmune disease & $1(2.3)$ & $4(9.1)$ & $.360^{\mathrm{b}}$ \\
\hline DM & $7(16.3)$ & $12(27.3)$ & $.300^{\mathrm{b}}$ \\
\hline \multicolumn{4}{|l|}{ Laboratory findings } \\
\hline Leukocyte count (PE) & $2456(1020-3500)$ & $1110(225-3420)$ & $.205^{c}$ \\
\hline ADA & $63(48-103)$ & $85(46-125)$ & $.228^{c}$ \\
\hline Albumin, $\mathrm{g} / \mathrm{dL}$ & $3.6 \pm 0.6$ & $3.3 \pm 0.7$ & $.047^{\mathrm{a}}$ \\
\hline Hypoalbuminemia, n (\%) & $7(16.3)$ & $17(38.6)$ & $.020^{\mathrm{b}}$ \\
\hline \multicolumn{4}{|l|}{ Symptoms } \\
\hline Dyspnea & $27(62.8)$ & $37(84.1)$ & $.024^{\mathrm{b}}$ \\
\hline Chest pain & $17(39.5)$ & $16(36.4)$ & $.761^{\mathrm{b}}$ \\
\hline Fever & $14(32.6)$ & $8(18.2)$ & $.123^{\mathrm{b}}$ \\
\hline Palpitation & $2(4.7)$ & $7(15.9)$ & $.157^{\mathrm{b}}$ \\
\hline Duration, days & $7(5-21)$ & $14(7-28)$ & $.433^{c}$ \\
\hline PE amount & & & $.023^{\mathrm{d}}$ \\
\hline Small & $8(18.6)$ & $4(9.1)$ & $.198^{\mathrm{b}}$ \\
\hline Moderate & $12(27.9)$ & $5(11.4)$ & $.062^{\mathrm{b}}$ \\
\hline Large & $23(53.5)$ & $35(79.5)$ & $.010^{\mathrm{b}}$ \\
\hline $\begin{array}{l}\text { Concomitant pulmonary } \\
\text { tuberculosis }\end{array}$ & $6(14.0)$ & $4(9.1)$ & $.521^{\mathrm{b}}$ \\
\hline \multicolumn{4}{|l|}{ Origin } \\
\hline Lung & $8(18.6)$ & $6(13.6)$ & $.528^{\mathrm{b}}$ \\
\hline Reactivation and inapparent & $35(81.4)$ & $38(86.4)$ & \\
\hline Hospital stay, days & $12.2 \pm 11.0$ & $14.3 \pm 10.5$ & $.348^{\mathrm{a}}$ \\
\hline \multicolumn{4}{|l|}{ Antituberculosis medication } \\
\hline Duration, months & $6.4 \pm 2.3$ & $6.6 \pm 3.3$ & $.776^{\mathrm{a}}$ \\
\hline \multicolumn{4}{|l|}{ Steroid } \\
\hline Users, yes (\%) & $19(44.2)$ & $22(50.0)$ & $.587^{\mathrm{b}}$ \\
\hline Duration, weeks & $12.1 \pm 7.4$ & $11.6 \pm 7.3$ & $.808^{\mathrm{a}}$ \\
\hline
\end{tabular}

Data are expressed as the mean \pm standard deviation or number (percentage) $B M I$ body mass index, $D M$ diabetes mellitus, $P E$ pericardial effusion, $A D A$ adenosine deaminase

${ }^{\text {a }}$ Student's $t$-test

'Pearson's $x$-test

'Mann-Whitney U-test, median (interquartile range)

${ }^{\mathrm{d}}$ Linear-by-linear association

edefined as serum albumin concentration below $3.0 \mathrm{~g} / \mathrm{dL}$ 
with unfavorable outcomes were admitted for hospital care earlier; however, the difference was not statistically significant.

All strains of M. tuberculosis isolated from the patients were antimicrobial susceptible strains in this study. Anti-TB medication (standard isoniazid, rifampin, ethambutol, and pyrazinamide for 2 months followed by maintenance 4 months of isoniazid, rifampin, and ethambutol) was taken for a duration of $6.4 \pm 2.3$ months in the favorable outcome group and $6.6 \pm 3.3$ months in the unfavorable outcome group, with no significant difference $(P=.776)$. Steroids were administered in $19(44.2 \%)$ patients with favorable outcomes and in $22(50 \%)$ patients with unfavorable outcomes $(P=.587)$. The mean duration of steroid use (prednisolone $60 \mathrm{mg} /$ day for four weeks, followed by $30 \mathrm{mg} /$ day for four weeks, $15 \mathrm{mg} /$ day for two weeks, and $5 \mathrm{mg} /$ day for 1 week [14]) was $12.1 \pm 7.4$ weeks in the favorable outcome group and $11.6 \pm 7.3$ weeks in the unfavorable outcome group $(P=.808)$. In the Kaplan Meier analysis, patients with cardiac tamponade had a lower rate of survival compared to patients with favorable outcomes (mean survival days: 1 day vs. 28 days, $P<.001$ ).

In the multivariate analysis, a large amount of pericardial effusion on echocardiogram (odds ratio [OR] 5.974, 95\% CI 1.811-19.703, $P=.003$ ), hypoalbuminemia (OR 4.905, 95\% CI 1.443-16.667, $P=.011$ ), and absence of underlying cardiovascular disease (OR $0.255,95 \%$ CI 0.089-0.733, $P=.011$ ) were shown to be independent predictive factors for unfavorable outcomes (Table 2).

\section{Discussion}

The infection of $M$. tuberculosis in the pericardium usually occurs through the retrograde lymphatic spread of the infection from the lungs, mediastinum structures, and adjacent lymph nodes, or through the hematogenous spread from the spine or from genitourinary infection [15]. The pathogenesis of TB pericarditis is a delayed hypersensitivity response induced by the activation of lymphocytes releasing lymphocytokines that activate macrophages, leading to granuloma formation and pericardial

Table 2 Multivariate analysis of predictive factors for unfavorable outcomes in patients with tuberculous pericarditis

\begin{tabular}{lll}
\hline Variables & OR $(95 \% \mathrm{Cl})$ & $P$-value \\
\hline Large PE & $5.974(1.811-19.703)$ & .003 \\
Cardiovascular disease & $0.255(0.089-0.733)$ & .011 \\
Hypoalbuminemia $^{\mathrm{a}}$ & $4.905(1.443-16.667)$ & .011 \\
Dyspnea & $2.792(0.852-9.145)$ & .090 \\
Male & $2.063(0.745-5.713)$ & .163 \\
\hline
\end{tabular}

$O R$ odds ratio, $C l$ confidence interval, $P E$ pericardial effusion

${ }^{a}$ defined as serum albumin concentration below $3.0 \mathrm{~g} / \mathrm{dL}$ effusion [16]. The histopathological pattern is mostly affected by the immune state, and HIV-infected persons with severely depleted CD4 lymphocytes present with less granuloma on biopsy [17].

While there is no difference in the sex-age pattern of the disease in industrialized countries, the disease rate in adult men exceeds those in women in developing countries [18]. The prevalence of $M$. tuberculosis infection in developing countries is similar to these findings, with a male predominance in patients older than 15 years [18]. The difference in the tuberculosis prevalence and sex is explained by the fact that women in developing countries have a passive attitude about visiting health-care facilities owing to cultural reasons [19]. The result of our study shows that male sex confers a higher risk for unfavorable outcomes.

The normal amount of pericardial fluid is $10-15 \mathrm{~mL}$ between the pericardial layers, and acts as a lubricant [20]. An inflammatory process of any cause results in an increased production of pericardial fluid [20, 21]. In previous studies, patients with hemodynamic compromise have large amounts of pericardial effusion on echocardiogram and are often associated with a higher mortality rate [2, 22]. Hemodynamically significant pericardial effusions leading to cardiac tamponade are commonly apparent with dyspnea $[6,23]$. Dyspnea on exertion is a key symptom of acute pericardial tamponade that requires emergency pericardiocentesis and has a high risk for mortality [24]. Choi et al. reported that patients who were initially more symptomatic, and had echogenic pericardial effusions tended to require pericardiectomy more often, and had more advanced disease [25]. From these results, it can be presumed that patients with large amounts of pericardial effusion are likely to experience dyspnea leading to hemodynamic compromise, which is correlated with unfavorable outcomes.

In this study, the presence of cardiovascular disease was a predictive factor for favorable outcomes. In this study, patients with an underlying cardiovascular disease were regularly being followed by the hospital, which may have resulted in the earlier detection of symptoms or provided the availability of earlier medical care, compared with patients who did not conduct regular hospital visits. For these reasons, patients with an underlying cardiovascular disease have a low risk for unfavorable outcomes. However, further studies for general applicability, and to confirm the validity of this assertion is needed.

In a study conducted by Reuter et al., patients uninfected with HIV had significantly higher serum protein and globulin levels than HIV-infected tuberculosis patients [8]. Although there was no statistical significance in serum albumin levels, the serum albumin/globulin 
ratios showed significantly lower results in the HIVuninfected TB pericarditis group [8]. One study in India identified serum albumin concentrations $<3.2 \mathrm{~g} / \mathrm{dL}$ as a risk factor for increased mortality in HIV-infected patients with tuberculosis [26]. However, there have been no studies on serum albumin level and its prognostic value in HIV-uninfected patients with TB pericarditis. The significance of this study is that it shows serum albumin as an independent variable predictive of unfavorable outcome in HIV-uninfected patients.

ADA is considered a marker for cellular immunity and is associated with the differentiation of lymphocytes [27]. A cutoff value of $40 \mathrm{U} / \mathrm{L}$ in the pericardial fluid is considered to have a specificity and sensitivity of $72 \%$ and $89 \%$ in diagnosis of TB pericarditis, respectively [28]. A median ADA of $97 \mathrm{U} / \mathrm{L}$ in the pericardial fluid was seen in the patients of this study.

The South Korean population receives routine Bacille Calmette-Guérin (BCG) vaccination after birth, and again at age 12 or 13 years if the child proves to be a tuberculin skin test (TST) nonresponder. Because BCG vaccination shows cross-reactivity with the result of the TST, we considered serum IGRA to be more appropriate than the TST for the diagnosis of latent tuberculosis [29]. In a study by Burgess et al., the measurement of pericardial fluid IGRA with a cutoff level of $>200 \mathrm{pg} / \mathrm{L}$ was highly specific (100\%) [30]. Future studies in larger sample numbers may confirm the use of pericardial effusion IGRA measurement as a promising method for the rapid diagnosis of TB pericarditis.

Anti-TB therapy reduces the incidence of mortality and constrictive pericarditis [7,31]. The treatment duration and regimen approach in HIV-uninfected patients is the same as those for pulmonary tuberculosis [32, 33]. The 6-month treatment regimen has similar effectiveness to that of longer treatment duration, and is generally recommended in susceptible tuberculosis cases [33]. Although the antimycobacterial treatment duration was slightly longer in the unfavorable group, it did not have any statistical difference with a median treatment duration of 6 months $(6.6 \pm 3.3$ vs. $6.4 \pm 2.3, P=.776)$.

In several prior studies, the use of corticosteroids significantly reduced the incidence of constrictive pericarditis, and thereby patients at high risk of progression to constrictive pericarditis are suggested to receive an adjunctive use of corticosteroids with a recommended starting dose of $60 \mathrm{mg} /$ day tapered during 11 weeks $[10,14,34]$. Another 10 years follow-up study by Strang et al. described that prednisolone reduced the risk of death due to pericarditis, and the need for repeat pericardiocentesis in patients with constrictive pericarditis or pericardial effusion [35, 36]. In our study, among the 40 patients with effusion or constrictive pericarditis, 21 were treated with steroids during a mean duration of 11 weeks but did not show any association to unfavorable outcomes. Hence, there was a difference in outcomes compared to previous clinical trials. However, these previous trials had relatively small samples, and a recent randomized trial of 1400 patients (431 whom were HIV-negative) by Mayosi et al. demonstrated similar results to our study, that routine use of adjuvant corticosteroids did not have any significant effect on the primary outcomes of death and cardiac tamponade [10].

This study has several limitations. First, this study was conducted retrospectively. Therefore, information on nutritional status, which could influence the treatment outcome, was not available. Second, only a small number of patients were included. Third, while it is plausible that routine follow up for cardiovascular disease may have enhanced earlier detection of symptoms, the explanation for this association may have limitations for general applicability. Finally, this study was performed in a country with an intermediate tuberculosis prevalence. Future multicenter prospective studies in countries with a diverse tuberculosis epidemiology are needed to confirm the predictive factors for unfavorable outcomes in HIVuninfected persons.

\section{Conclusion}

HIV-uninfected patients with TB pericarditis are at a higher risk for unfavorable outcomes when presenting with hypoalbuminemia, with large pericardial effusion, and without cardiovascular disease. This study may prompt clinicians to perform early diagnosis and treatment in patients with these risk factors, which may lead to a decrease in mortality and late complications such as constrictive pericarditis.

\section{Additional file}

Additional file 1: Table S1. Definition of diagnostic categories. (DOCX $21 \mathrm{~kb}$ )

\section{Abbreviations}

ADA: Adenosine deaminase; BCG: Bacille Calmette-Guérin; Cl: Confidence interval; HIV: Human immunodeficiency virus; IFN- - : Interferon-gamma; IGRA: Interferon gamma release assay; OR: Odds ratio; RBC: Red blood cell; TB: Tuberculous; TST: Tuberculin skin test

\section{Acknowledgements}

Not applicable.

\section{Funding}

No funding was received for this study

Availability of data and materials

All data generated or analysed during this study are included in this published article and its supplementary/additional information files. However raw dataset are available from the corresponding author on reasonable request. 


\section{Authors' contributions}

IJY designed the study and acquired data, analyzed and interpreted the data, drafted the initial manuscript, reviewed, and critically revised and approved the final manuscript as submitted. SJS conceptualized the study and is responsible for the content of the manuscript, including the data and analysis. YGS, JYC, MHK, WYJ, DHO, YCK, JES, EJK, JUL, NSK, and JMK provided statistical assistance and revised and approved the final manuscript. All authors read and approved the final manuscript.

\section{Competing interests}

The authors declare that they have no competing interests.

\section{Consent for publication}

Not applicable.

\section{Ethics approval and consent to participate}

Not applicable.

Received: 16 July 2016 Accepted: 23 November 2016

Published online: 29 November 2016

\section{References}

1. Dheda K, Barry 3rd CE, Maartens G. Tuberculosis. Lancet (London, England). 2016;387(10024):1211-26.

2. Ntsekhe M, Mayosi BM. Tuberculous pericarditis with and without HIV. Heart Fail Rev. 2013:18(3):367-73.

3. Trautner BW, Darouiche RO. Tuberculous pericarditis: optimal diagnosis and management. Clin Infect Dis. 2001;33(7):954-61.

4. Cegielski JP, Lwakatare J, Dukes CS, Lema LE, Lallinger GJ, Kitinya J, Reller LB, Sheriff F. Tuberculous pericarditis in Tanzanian patients with and without HIV infection. Tuber Lung Dis. 1994;75(6):429-34.

5. American Thoracic Society. Diagnostic Standards and Classification of Tuberculosis in Adults and Children. Am J Respir Crit Care Med. 2000;161(4 Pt 1): 1376-95

6. Fowler NO, Manitsas GT. Infectious pericarditis. Prog Cardiovasc Dis. 1973;16(3):323-36

7. Gooi HC, Smith JM. Tuberculous pericarditis in Birmingham. Thorax. 1978;33(1):94-6.

8. Reuter $H$, Burgess $L$, van Vuuren W, Doubell A. Diagnosing tuberculous pericarditis. QJM. 2006;99(12):827-39.

9. Bhan GL. Tuberculous pericarditis. J Infect. 1980;2(4):360-4.

10. Mayosi BM, Ntsekhe M, Bosch J, Pandie S, Jung H, Gumedze F, Pogue J, Thabane L, Smieja M, Francis V, et al. Prednisolone and Mycobacterium indicus pranii in tuberculous pericarditis. N Engl J Med. 2014;371(12):1121-30.

11. Adler Y, Charron P, Imazio M, Badano L, Baron-Esquivias G, Bogaert J, Brucato A, Gueret P, Klingel K, Lionis C, et al. 2015 ESC Guidelines for the diagnosis and management of pericardial diseases: the task force for the diagnosis and management of pericardial diseases of the European Society of Cardiology (ESC) Endorsed by: The European Association for CardioThoracic Surgery (EACTS). Eur Heart J. 2015;36(42):2921-64.

12. Tobias DK, Pan A, Jackson CL, O'Reilly EJ, Ding EL, Willett WC, Manson JE, Hu FB. Body-mass index and mortality among adults with incident type 2 diabetes. N Engl J Med. 2014;370(3):233-44.

13. McClure S, Custer E, Bessman JD. Improved detection of early iron deficiency in nonanemic subjects. JAMA. 1985;253(7):1021-3.

14. Centers for Disease Control and Prevention.Treatment of tuberculosis, American Thoracic Society, CDC, and Infectious Diseases Society of America. MMWR. 2003; 52(Rr-11):1-77.

15. Mayosi BM, Burgess $\perp$, Doubell AF. Tuberculous pericarditis. Circulation. 2005;112(23):3608-16.

16. Agner RC, Gallis HA. Pericarditis: differential diagnostic considerations. Arch Intern Med. 1979;139(4):407-12.

17. Reuter H, Burgess $L$, Schneider J, Van Vuuren W, Doubell AF. The role of histopathology in establishing the diagnosis of tuberculous pericardial effusions in the presence of HIV. Histopathology. 2006;48(3):295-302

18. Holmes CB, Hausler $H$, Nunn P. A review of sex differences in the epidemiology of tuberculosis. Int J Tuberc Lung Dis. 1998;2(2):96-104.

19. Neyrolles O, Quintana-Murci L. Sexual inequality in tuberculosis. PLoS Med. 2009;6(12):e1000199.

20. Imazio $M$, Adler Y. Management of pericardial effusion. Eur Heart J. 2013;34(16):1186-97.
21. Corey GR, Campbell PT, Van Trigt P, Kenney RT, O'Connor CM, Sheikh KH, Kisslo JA, Wall TC. Etiology of large pericardial effusions. Am J Med. 1993;95(2):209-13.

22. Ortbals DW, Avioli LV. Tuberculous pericarditis. Arch Intern Med. 1979;139(2):231-4

23. Permanyer-Miralda G. Acute pericardial disease: approach to the aetiologic diagnosis. Heart (British Cardiac Society). 2004;90(3):252-4.

24. Spodick DH. Acute cardiac tamponade. N Engl J Med. 2003;349(7):684-90

25. Choi HO, Song JM, Shim TS, Kim SH, Jung IH, Kang DH, Song JK. Prognostic value of initial echocardiographic features in patients with tuberculous pericarditis. Korean Circ J. 2010;40(8):377-86.

26. Alvarez-Uria G, Midde M, Pakam R, Naik PK. Diagnostic and prognostic value of serum albumin for tuberculosis in HIV infected patients eligible for antiretroviral therapy: data from an HIV cohort study in India. Bioimpacts. 2013:3(3):123-8.

27. Blake J, Berman P. The use of adenosine deaminase assays in the diagnosis of tuberculosis. S Afr Med J. 1982;62(1):19-21.

28. Tuon FF, Silva VI, Almeida GM, Antonangelo L, Ho YL. The usefulness of adenosine deaminase in the diagnosis of tuberculous pericarditis. Rev Inst Med Trop Sao Paulo. 2007:49(3):165-70.

29. Farhat M, Greenaway C, Pai M, Menzies D. False-positive tuberculin skin tests: what is the absolute effect of BCG and non-tuberculous mycobacteria? Int J Tuberc Lung Dis. 2006;10(11):1192-204.

30. Burgess LJ, Reuter H, Carstens ME, Taljaard JJ, Doubell AF. The use of adenosine deaminase and interferon-gamma as diagnostic tools for tuberculous pericarditis. Chest. 2002;122(3):900-5.

31. Long R, Younes M, Patton N, Hershfield E. Tuberculous pericarditis: longterm outcome in patients who received medical therapy alone. Am Heart J. 1989;117(5):1133-9.

32. Small PM, Fujiwara PI. Management of tuberculosis in the United States. N Engl J Med. 2001;345(3):189-200.

33. Combs DL, O'Brien RJ, Geiter LJ. USPHS tuberculosis short-course chemotherapy trial 21: effectiveness, toxicity, and acceptability. The report of final results. Ann Intern Med. 1990;112(6):397-406.

34. Hakim JG, Ternouth I, Mushangi E, Siziya S, Robertson V, Malin A. Double blind randomised placebo controlled trial of adjunctive prednisolone in the treatment of effusive tuberculous pericarditis in HIV seropositive patients. Heart (British Cardiac Society). 2000;84(2):183-8.

35. Strang JI, Nunn AJ, Johnson DA, Casbard A, Gibson DG, Girling DJ. Management of tuberculous constrictive pericarditis and tuberculous pericardial effusion in Transkei: results at 10 years follow-up. QJM. 2004:97(8):525-35.

36. Strang Jl, Kakaza HH, Gibson DG, Allen BW, Mitchison DA, Evans DJ, Girling DJ, Nunn AJ, Fox W. Controlled clinical trial of complete open surgical drainage and of prednisolone in treatment of tuberculous pericardial effusion in Transkei. Lancet (London, England). 1988;2(8614):759-64.

\section{Submit your next manuscript to BioMed Central and we will help you at every step:}

- We accept pre-submission inquiries

- Our selector tool helps you to find the most relevant journal

- We provide round the clock customer support

- Convenient online submission

- Thorough peer review

- Inclusion in PubMed and all major indexing services

- Maximum visibility for your research

Submit your manuscript at www.biomedcentral.com/submit 